\title{
DC dielectrophoretic focusing of particles in a serpentine microchannel
}

\author{
Junjie Zhu • Tzuen-Rong J. Tzeng • \\ Guoqing $\mathrm{Hu} \cdot$ Xiangchun Xuan
}

Received: 5 January 2009/Accepted: 6 March 2009/Published online: 10 April 2009

(C) Springer-Verlag 2009

\begin{abstract}
Focusing particles into a tight stream is usually a necessary step prior to separating and sorting them. We present herein a proof-of-concept experiment of a novel particle focusing technique in DC electrokinetic flow through a planar serpentine microchannel. This focusing stems from the cross-stream dielectrophoretic motion induced within the channel turns. The observed particle focusing behavior is consistent with the predicted particle trajectories from a numerical modeling.
\end{abstract}

Keywords Particle focusing - DC dielectrophoresis . Electrokinetic flow $\cdot$ Serpentine microchannel

\section{Introduction}

Focusing particles (both biological and synthetic) into a tight stream is usually a necessary step prior to separating and sorting them (Huh et al. 2005; Chung and Kim 2007; Pamme 2007; Ateya et al. 2008). Particle focusing may be realized by using hydrodynamic (Lee et al. 2006; Simonnet and Groisman 2006; Chang et al. 2007; Tsai et al. 2008) or electrokinetic (Fu et al. 2003; Yang et al. 2005; Xuan and

J. Zhu $\cdot$ X. Xuan $(\bowtie)$

Department of Mechanical Engineering, Clemson University, Clemson, SC 29634-0921, USA

e-mail: xcxuan@clemson.edu

T.-R. J. Tzeng

Department of Biological Sciences, Clemson University, Clemson, SC 29634-0314, USA

G. $\mathrm{Hu}$

LNM, Institute of Mechanics, Chinese Academy of Sciences, 100190 Beijing, China
Li 2005; Kohlheyer et al. 2008) sheath flows to pinch the suspending medium and thus focus particles. This approach, however, requires a precise control of the flow rate of both the sheath flows and the particle stream. Particle focusing may also be achieved by applying an external force field, e.g., optical (Zhao et al. 2007), acoustic (Shi et al. 2008), electrophoretic (Takahashi et al. 2003), or AC dielectrophoretic (Lin et al. 2004; Yu et al. 2005; Cheng et al. 2007; Tornay et al. 2008), to manipulate particles directly to their equilibrium positions. This approach, however, requires an additional pressure-pumping of the particle stream, not to mention the extra set ups for generating the external forces. Moreover, the particle throughput is often limited as the time for forces to act on particles decreases with increasing flow rate.

The concurrent pumping of the particle stream and focusing of particles have been realized by DC electrokinetic flow via the induced dielectrophoretic particle motion. However, an array or pairs of insulative microstructures such as oil menisci (Cummings and Singh 2003; Xuan et al. 2006; Thwar et al. 2007) are necessary parts within the microchannel in order to create the non-uniform electric field. Moreover, the generally strong electric field, shear stress and Joule heating in the constrictions formed by the microstructures may pose significant problems to cell viability (Voldman 2006). Another approach to focusing particles is based on particle hydrophoresis that is generated by the lateral pressure gradient induced in a channel comprising an array of obstacles on the top and bottom walls (Choi et al. 2007; Choi and Park 2008). This approach is, however, sensitive to the structure of the obstacles and requires a non-trivial fabrication. Recently, inertia has also been exploited to implement a continuous focusing of particles in curved microchannels (Di Carlo et al. 2007, 2008; Mao et al. 2007; Seo et al. 2007a, b). The 
equilibrium position of the focused particle stream is, however, dependent on the Reynolds number. Moreover, particles may become defocused when the Reynolds number is above one threshold value.

In this paper, we introduce a novel particle focusing technique in DC electrokinetic flow through a planar serpentine microchannel with constant width and depth. This focusing stems from the cross-stream dielectrophoretic motion of particles induced within the channel turns. Neither an extra pressure-pumping nor embedded microstructures are required in this DC dielectrophoretic focusing of particles.

\section{Mechanism}

Figure 1 shows the electric field lines (E, with arrows indicating the direction) and the contour of electric field intensity (the darker the higher) in one s-shaped period of a serpentine microchannel, i.e., a left U-turn immediately followed by a right U-turn. It is apparent that the electric field becomes non-uniform and attains the maximum and minimum values at the inner and outer corners of each of the four $90^{\circ}$ turns. Hence, particles experience a dielectrophoretic force, $\mathbf{F}_{\text {DEP }}$ (bold symbol denotes a vector hereafter) when they move electrokinetically through a channel turn. In DC electric fields, $\mathbf{F}_{\mathrm{DEP}}$ on an isolated spherical particle is given by (Morgan and Green 2002)

$$
\begin{gathered}
\mathbf{F}_{\mathrm{DEP}}=(1 / 2) \pi \varepsilon_{\mathrm{f}} d^{3} f_{\mathrm{CM}}(\mathbf{E} \cdot \nabla \mathbf{E}) \quad \text { and } \\
f_{\mathrm{CM}}=\left(\sigma_{\mathrm{p}}-\sigma_{\mathrm{f}}\right) /\left(\sigma_{\mathrm{p}}+2 \sigma_{\mathrm{f}}\right),
\end{gathered}
$$

where $\varepsilon_{\mathrm{f}}$ is the fluid permittivity, $d$ the particle diameter, $f_{\mathrm{CM}}$ the so-called Clausius-Mossotti (CM) factor, $\mathbf{E}$ the

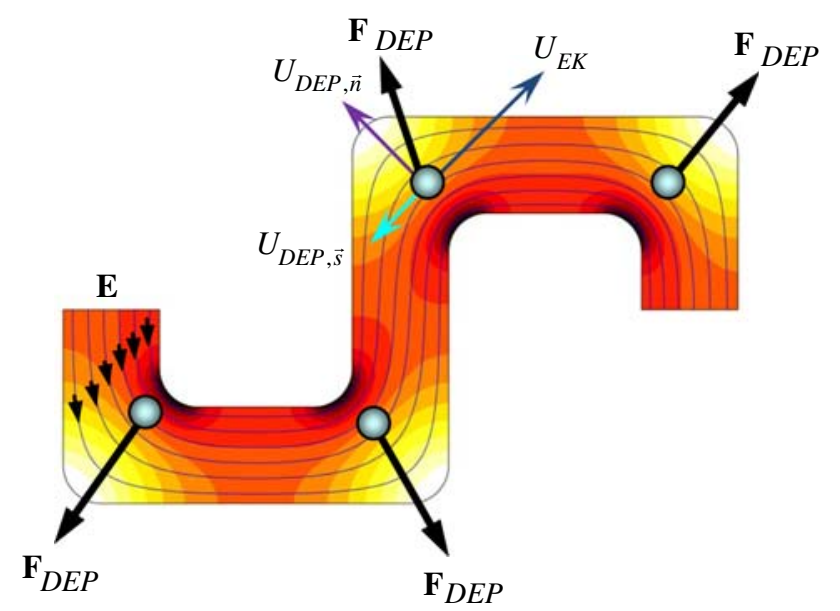

Fig. 1 Mechanism of the DC dielectrophoretic focusing of particles in a serpentine microchannel (only one s-shaped period is exhibited). Both the induced dielectrophoretic force in each turn and the particle velocity components in streamline coordinates are illustrated. The background shows the contour of electric field intensity (the darker the higher). The arrowed curves represent the electric field lines (E) electric field vector, $\sigma_{\mathrm{p}}$ the electric conductivity of particles, and $\sigma_{\mathrm{f}}$ the electric conductivity of the suspending fluid. Note that Eq. 1 is valid only for weak electrolyte solutions (Shilov et al. 2000) and small particles (more accurately, the size of the particle is much smaller than the characteristic length scale of the electric field) (Morgan and Green 2002).

As polystyrene particles and live biological cells appear insulating in DC and low-frequency AC electric fields $(<100 \mathrm{kHz}$ ) (Ermolina and Morgan 2005; Voldman 2006), we generally have $\sigma_{\mathrm{p}}<\sigma_{\mathrm{f}}$ and so $f_{\mathrm{CM}}<0$ leading to negative dielectrophoresis. In other words, $\mathbf{F}_{\mathrm{DEP}}$ is directed toward the lower electric field region as indicated in Fig. 1. Therefore, particles will migrate across streamlines from the inner corner to the outer corner in each of the turns. As a matter of fact, the streamlines are equivalent to the electric field lines illustrated in Fig. 1 due to the similarity between flow and electric fields in pure electrokinetic flows (Santiago 2001). Since the inner and outer corners switch between the left and right U-turns, particles will experience $\mathbf{F}_{\text {DEP }}$ of alternate directions within the turns. Moreover, as $\mathbf{F}_{\text {DEP }}$ is always stronger in the inner corner than in the outer corner, particles tend to be deflected toward the channel center region in each of the serpentine period (see Fig. 1). The overall consequence will thus be a focused particle stream along the channel centerline.

By balancing $\mathbf{F}_{\text {DEP }}$ and the electrophoretic force with the Stokes drag force, one can obtain the net particle velocity, $\mathbf{U}_{\mathrm{p}}$, as

$$
\begin{aligned}
& \mathbf{U}_{\mathrm{p}}=\mathbf{U}_{\mathrm{EK}}+\mathbf{U}_{\mathrm{DEP}}=\mu_{\mathrm{EK}} \mathbf{E}+\mu_{\mathrm{DEP}}(\mathbf{E} \cdot \nabla \mathbf{E}) \quad \text { and } \\
& \mu_{\mathrm{DEP}}=\varepsilon_{\mathrm{f}} d^{2} f_{\mathrm{CM}} / 6 \mu_{\mathrm{f}},
\end{aligned}
$$

which is composed of the electrokinetic motion (a combination of fluid electroosmosis and particle electrophoresis), $\mathbf{U}_{\mathrm{EK}}$, and the dielectrophoretic motion, $\mathbf{U}_{\mathrm{DEP}}$. In the last equation, $\mu_{\mathrm{EK}}$ is the electrokinetic mobility, $\mu_{\mathrm{DEP}}$ the dielectrophoretic mobility which is negative as $f_{\mathrm{CM}}<0$, and $\mu_{\mathrm{f}}$ the fluid viscosity. Note that the particle and fluid inertial motions have been neglected in Eq. 2 as the Reynolds and Dean numbers are both small $(\ll 1)$ under the experimental conditions (Di Carlo et al. 2007, 2008). As the particle focusing is attributed to the cross-stream dielectrophoretic motion, we may conveniently express the particle velocity, $\mathbf{U}_{\mathrm{p}}$, in terms of streamline coordinates (see Fig. 1),

$$
\begin{aligned}
\mathbf{U}_{\mathrm{p}} & =\left(U_{\mathrm{DEP}, \vec{s}}+U_{\mathrm{EK}}\right) \hat{\mathbf{s}}+U_{\mathrm{DEP}, \vec{n}} \hat{\mathbf{n}} \\
& =\left(\mu_{\mathrm{DEP}} E \frac{\partial E}{\partial s}+\mu_{\mathrm{EK}} E\right) \hat{\mathbf{s}}+\mu_{\mathrm{DEP}} \frac{E^{2}}{\Re} \hat{\mathbf{n}},
\end{aligned}
$$

where $U_{\mathrm{DEP}, \vec{s}}$ is the dielectrophoretic particle velocity in the streamline direction with the unit vector $\hat{\mathbf{s}}, U_{\mathrm{EK}}$ the streamwise electrokinetic velocity, $U_{\mathrm{DEP}, \vec{n}}$ the dielectrophoretic particle velocity normal to the streamline direction with the unit vector $\hat{\mathbf{n}}, E$ the electric field intensity, and $\Re$ 
the radius of curvature of the streamline which should follow closely the turn radius when fluid inertia is negligible.

The efficiency of the DC dielectrophoretic focusing of particles in a serpentine microchannel is determined by the ratio of the distance a particle moves perpendicular to the streamline to the distance travelled along the streamline. This ratio, the larger the better focusing, is equivalent to the ratio of particle velocity perpendicular and parallel to the streamline, i.e., $U_{\mathrm{DEP}, \vec{n}} /\left(U_{\mathrm{DEP}, \vec{s}}+U_{\mathrm{EK}}\right)$ as seen in Eq. 3, which may be approximated as

$\frac{U_{\mathrm{DEP}, \vec{n}}}{U_{\mathrm{EK}}}=\left(\frac{\mu_{\mathrm{DEP}}}{\mu_{\mathrm{EK}}}\right) \frac{E}{\Re}$

where $U_{\mathrm{DEP}, \vec{s}}$ has been assumed to have a much smaller magnitude than $U_{\mathrm{EK}}$. This assumption is generally valid in microchannels with a constant width unless $U_{\mathrm{EK}}$ is trivial due to the counter-balanced particle electrophoresis and fluid electroosmosis. Hence, a larger electric field and/or a smaller turn radius should provide a better focusing. However, it is important to note that a microchannel turn with a very small radius, i.e., a sharp turn, may induce a non-linear electrokinetic flow due to the electric field leakage (Thamida and Chang 2002), which is believed to affect the particle focusing performance. In addition, as $\mu_{\text {DEP }}$ is proportional to the particle diameter squared (see Eq. 2) while $\mu_{\mathrm{EK}}$ is only a weak function of particle size (Anderson 1989), the dielectrophoretic focusing should work more efficiently for larger particles.

\section{Experiment}

Figure 2 displays a picture of the serpentine microchannel used in our experiments. It was fabricated in polydimethylsiloxane (PDMS) using the soft lithography technique (Duffy et al. 1998). The detailed procedure was given elsewhere (Xuan et al. 2005). The channel comprises a $1 \mathrm{~cm}$ long serpentine section between two $0.5 \mathrm{~cm}$ long straight sections. It has a uniform depth of $25 \mu \mathrm{m}$ as calculated from the speed of photoresist spinning. The inset in Fig. 2 shows the close-up view of the curved part. The channel is $50 \mu \mathrm{m}$ wide. The width and height of every U-turn are both $200 \mu \mathrm{m}$. Original solutions of polystyrene particles of 5 and $10 \mu \mathrm{m}$ in diameter (Sigma-Aldrich, St. Louis, MO) were re-suspended in $1 \mathrm{mM} \mathrm{KCl}$ at a volume ration of 1:50 and 1:20, respectively. The electric field was supplied by a DC power supply (Glassman High Voltage Inc., High Bridge, NJ). Particle transport through the serpentine channel was visualized through an inverted microscope (Nikon TE2000-U) equipped with a CCD camera (Nikon DS Qi1MC).

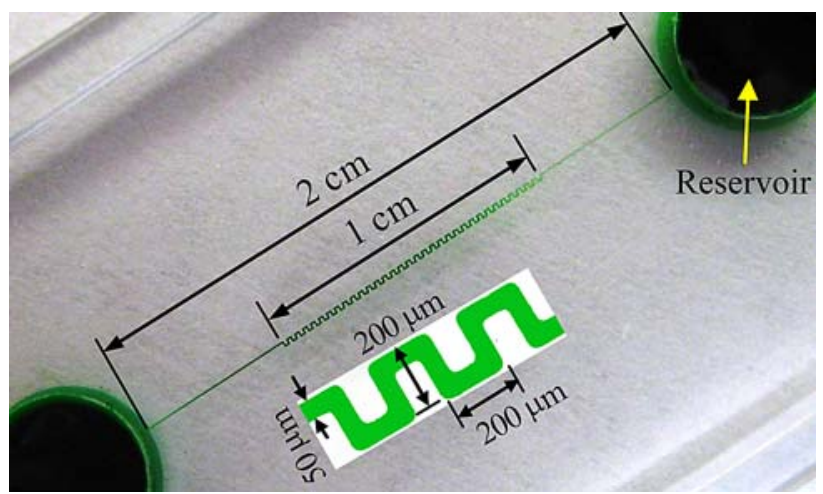

Fig. 2 Picture of the serpentine microchannel used in the experiment with dimensions indicated in the inset

\section{Modeling}

In order to understand and predict the observed particle focusing behavior, we developed a numerical model to simulate the electric field-mediated particle transport through the serpentine microchannel. This model is based on that developed by Kang et al. (2006a, b). For simplicity, the perturbations of particles on the flow and electric fields (via moving boundaries in both fields due to the finite particle size) were both neglected in our model, so were the particle-particle interactions. Instead, a correction factor $c$ was introduced to account for the effects of particle size (and others if any) on the dielectrophoretic velocity. As such, the particle velocity in Eq. 2 is revised as

$\mathbf{U}_{\mathrm{p}}=\mu_{\mathrm{EK}} \mathbf{E}+c \mu_{\mathrm{DEP}}(\mathbf{E} \cdot \nabla \mathbf{E})$.

This velocity was used in a particle tracing function in COMSOL (Burlington, MA) to compute the particle trajectory. Particles were assumed massless and uniformly distributed at the channel inlet. As particle dielectrophoresis happens only in the channel width direction, a 2D model was employed to predict the particle focusing without considering the effects of top and bottom channel walls on particle motion. This treatment has been proved reasonable in our recent studies (Kang et al. 2006a, b).

In our numerical model, the electrokinetic mobility, $\mu_{\mathrm{EK}}$, was obtained by measuring the average particle velocity in the straight section of the microchannel, which is $5.5 \times 10^{-8} \mathrm{~m}^{2} /(\mathrm{V} \mathrm{s})$ for both 5 and $10 \mu \mathrm{m}$ particles with an error of $10 \%$. The dielectrophoretic mobility, $\mu_{\mathrm{DEP}}$, was calculated from Eq. 2 with the typical dynamic viscosity, $\mu=0.9 \times 10^{-3} \mathrm{~kg} /(\mathrm{m} \mathrm{s})$ and permittivity $\varepsilon_{\mathrm{f}}=6.9 \times$ $10^{-10} \mathrm{C} /(\mathrm{v} \mathrm{m})$ for pure water at $25^{\circ} \mathrm{C}$. The calculated electric conductivities of 5 and $10 \mu \mathrm{m}$ particles, i.e., $\sigma_{\mathrm{p}}$ in Eq. 1, are 8.96 and $4.48 \mu \mathrm{S} / \mathrm{cm}$, respectively, if the surface conductance is assumed to be $1 \mathrm{nS}$ as suggested by Ermolina and Morgan (2005). The measured electric 
conductivity of the solution, i.e., $\sigma_{\mathrm{f}}$ in Eq. 1, is about $160 \mu \mathrm{S} / \mathrm{cm}$. Therefore, the $\mathrm{CM}$ factor, i.e., $f_{\mathrm{CM}}$ as defined in Eq. 1, was determined as -0.46 and -0.48 for 5 and $10 \mu \mathrm{m}$ particles, respectively. Under the assumption of a uniform electric conductivity of the suspending fluid, the electric field $\mathbf{E}=-\nabla \phi$ was computed by solving the Laplace equation $\nabla^{2} \phi=0$. The boundary conditions include the voltage drop between the channel ends and the insulating condition on the channel walls. The correction factor $c$ was determined by fitting the predicted particle trajectories to the width of the observed particle stream at the exit of the serpentine section.

\section{Results and discussion}

Figure $3 \mathrm{a}$ illustrates the experimentally obtained streak images of $5 \mu \mathrm{m}$ particles in the entrance (top) and exit (middle and bottom) regions of the serpentine section of the microchannel. The applied electric potential drops across the entire channel ( $2 \mathrm{~cm}$ straight length) are 200 and $400 \mathrm{~V}$ for the middle and bottom images, corresponding to a nominal electric field of 10 and $20 \mathrm{kV} / \mathrm{m}$ (the true values are 7.7 and $15.4 \mathrm{kV} / \mathrm{m}$ in the straight section), respectively. At both electric fields, particles are almost uniformly distributed prior to entering the serpentine section (top), indicating a zero-focusing effect in the straight section of the channel. In the exit region of the serpentine section, however, particles are moving in a focused stream along the channel centerline as expected. Moreover, the width of the focused particle stream decreases with the rise of electric field as noted above. It is estimated that over 1,200 particles can be dielectrophoretically focused within one minute in this serpentine microchannel at the electric field of $20 \mathrm{kV} / \mathrm{m}$. (a) Experiment
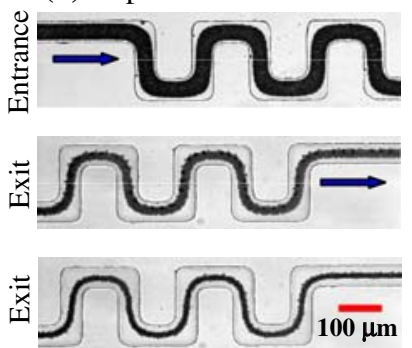

(b) Simulation
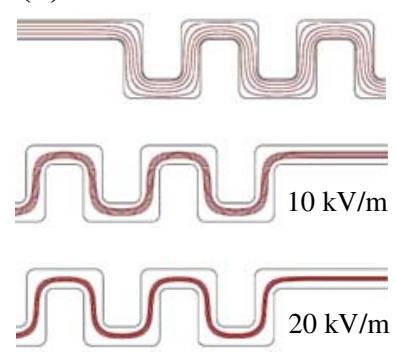

Fig. 3 Demonstration of the DC dielectrophoretic focusing of $5 \mu \mathrm{m}$ particles in a serpentine microchannel at different electric fields: a experimentally observed particle streak images, and b numerically predicted particle trajectories in the entrance (top) and exit (middle and bottom with electric fields indicated) regions of the serpentine section of the microchannel. The block arrows indicate the flow direction
Figure $3 \mathrm{~b}$ shows the numerically predicted trajectories of $5 \mu \mathrm{m}$ particles in the serpentine microchannel at the same conditions as those in the experiments. The correction factor $c$ was set to 0.5 . Close agreements between the experimentally and numerically obtained widths of the focused particle stream were found for both electric fields. Using this model, we have also extracted the width of $5 \mu \mathrm{m}$ particle stream with respect to the number of serpentine periods (refer to Fig. 1) that particles have passed through. The applied electric field was $20 \mathrm{kV} / \mathrm{m}$. As illustrated in Fig. 4, the initially uniformly distributed particles (with an assumed width of $50 \mu \mathrm{m}$ ) get focused as they migrate electrokinetically through the serpentine channel. Moreover, the focusing performance decreases along the channel (reflected by the decreasing slope of the illustrated curve in Fig. 4) because the overall cross-stream dielectrophoretic motion in one serpentine period becomes smaller when particles move closer to the centerline.

Figure 5a compares the streak images of $5 \mu \mathrm{m}$ (top) and $10 \mu \mathrm{m}$ (bottom) particles in the exit region of the serpentine section of the microchannel at a nominal electric field of $10 \mathrm{kV} / \mathrm{m}$. The measured width of the focused $10 \mu \mathrm{m}$ particle stream is $13 \mu \mathrm{m}$, which is as expected smaller than that for $5 \mu \mathrm{m}$ particles (the measured width is about $23 \mu \mathrm{m})$. The numerically predicted trajectories of $10 \mu \mathrm{m}$ particles in the serpentine microchannel at the experimental condition are displayed in Fig. 5b (bottom). The correction factor $c$ was set to 0.3 for the best fit to the measured width of the focused particle stream. While $c$ has been found insensitive to the applied electric field for both 5 and $10 \mu \mathrm{m}$ particles, it is still uncertain whether $c$ varies with the particle concentration and the channel structure, etc. We are currently examining this issue.

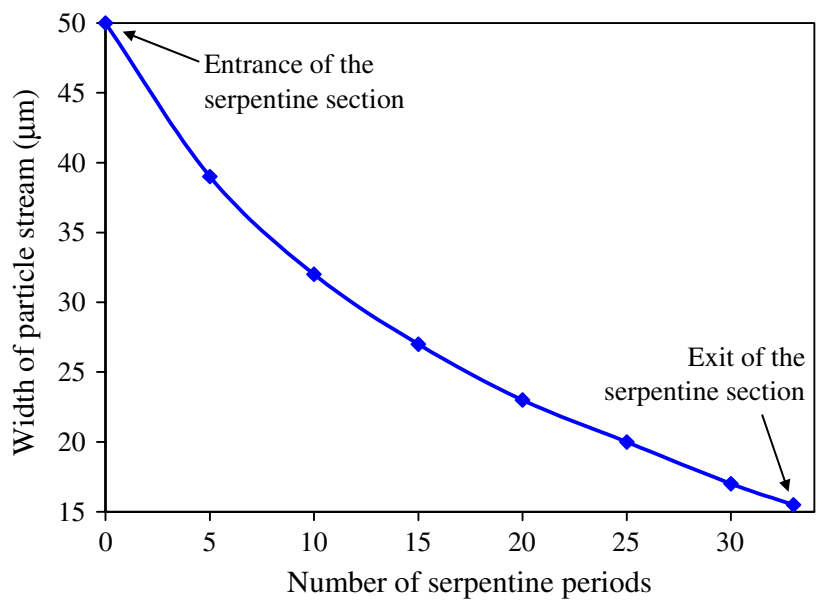

Fig. 4 Illustration of the $5 \mu \mathrm{m}$ particle stream width with respect to the number of serpentine periods (refer to Fig. 1) that particles have passed through. The applied electric field is $20 \mathrm{kV} / \mathrm{m}$ 
(a) Experiment

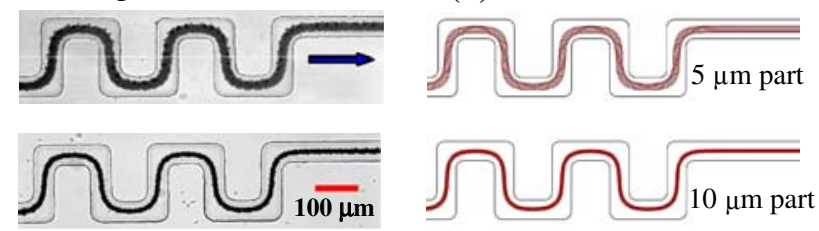

Fig. 5 Comparison of a experimentally observed streak images and $\mathbf{b}$ numerically predicted trajectories of $5 \mu \mathrm{m}$ (top) and $10 \mu \mathrm{m}$ (bottom) particles in the exit region of the serpentine section of the microchannel at a nominal electric field of $10 \mathrm{kV} / \mathrm{m}$. The block arrow indicates the flow direction

Besides the ratio of particle velocity perpendicular and parallel to the streamline as given in Eq. 4, the ultimate width of the focused particle stream in a serpentine microchannel is also dependent on the channel width and length (the total channel length in the serpentine section). In order to be focused to the channel centerline, all particles should have sufficient times to traverse at least half of the channel width before traveling out of the serpentine section. Therefore, reducing the width and increasing the length of the serpentine section should both improve the DC dielectrophoretic focusing of particles. More accurately, the latter condition should be replaced by the number of serpentine periods because particle dielectrophoresis only takes effects within the channel turns and the straight channels between them contribute little to focusing. It is also beneficial to use an AC electric field to generate the cross-stream dielectrophoretic particle motion while the pumping of the particle stream may be realized by a DC electric field or pressure gradient. We note that DC-biased AC electric fields have been recently employed to improve the dielectrophoretic separation of particles in structured microchannels (Hawkins et al. 2007; Lewpiriyawong et al. 2008).

If a very high DC electric field or a DC-biased AC field with a very small DC component is applied, the induced cross-stream dielectrophoretic motion may become dominant over the streamwise electrokinetic motion. As a consequence, particles will be quickly focused in a serpentine microchannel. This is supported by our numerical modeling of $5 \mu \mathrm{m}$ particle trajectories at high DC electric fields (i.e., 100, 200 and $500 \mathrm{kV} / \mathrm{m}$ ) as illustrated in Fig. 6. The particle, solution and channel properties were all assigned the same values as those used in the above validated simulations. It is apparent that particles are already well focused after passing through the first 2-3 serpentine periods at the electric field of $500 \mathrm{kV} / \mathrm{m}$. However, the focused particle stream does not flow along the channel centerline as observed at small electric fields (see Figs. 3 and 5), but meanders across the fluid stream in the

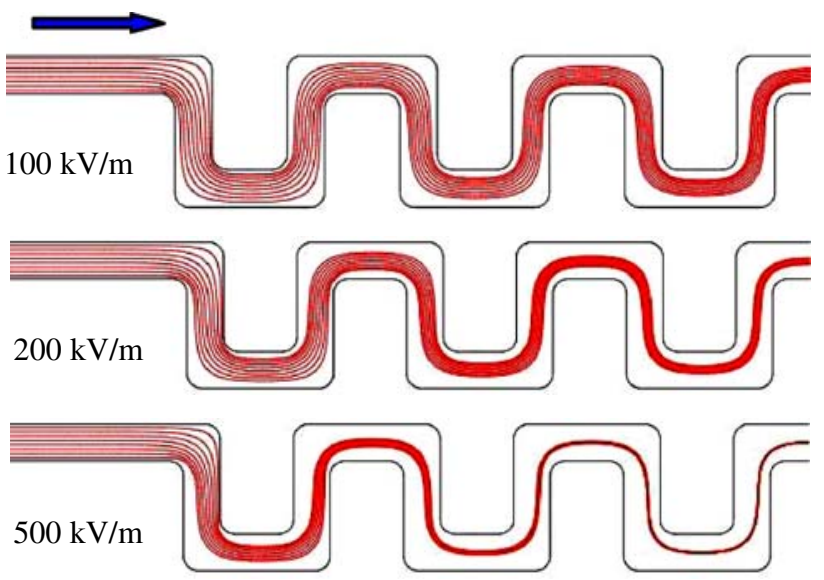

Fig. 6 Comparison of numerically predicted trajectories of $5 \mu \mathrm{m}$ particles in the entrance region of the serpentine section at different high DC electric fields (assumed reasonable). The block arrow indicates the flow direction in all three circumstances

serpentine section. As the electric field of hundreds of $\mathrm{kV} /$ $\mathrm{m}$ is rarely used in typical microfluidic applications, we will test the predictions in Fig. 6 in future experiments using a DC-biased AC electric field with very small DC components. For example, our model confirms that the particle focusing performance at the $500 \mathrm{kV} / \mathrm{m} \mathrm{DC}$ field is comparable to that at a $20 \mathrm{kV} / \mathrm{m}$ DC-biased AC field with the DC to AC field ratio being set to 0.04 .

\section{Conclusions}

We have demonstrated proof-of-concept of a novel particle focusing technique based on the cross-stream dielectrophoretic particle motion in the turns of a serpentine microchannel. The favoring of large electric fields makes this focusing technique very appealing as the efficiency and the particle throughput may be enhanced simultaneously. Moreover, the footprint of a serpentine microchannel can be readily made very small to save space for other integrated functionalities on microfluidic chips. This DC dielectrophoretic particle focusing technique is superior to the existing $\mathrm{AC}$ dielectrophoretic focusing technique in that both the in-channel microelectrodes and the external pumping force are eliminated. Hence, the fabrication and operational costs are reduced. We envision direct near-term applications of this novel focusing technique in continuous bioparticle separation and flow cytometry for a wide range of technological solutions in biology, medicine and industry.

Acknowledgments The support from Clemson University through a start-up package and a Research Equipment Fund to Xuan is gratefully acknowledged. The support from the State Key Laboratory of Nonlinear Mechanics in China is also gratefully appreciated. 


\section{References}

Anderson JL (1989) Colloidal transport by interfacial forces. Annu Rev Fluid Mech 21:61-99

Ateya DA, Erickson JS, Howell PB Jr, Hilliard LR et al (2008) The good, the bad, the tiny: a review of microflow cytometry. Anal Bioanal Chem 391:1485-1498

Chang CC, Huang ZY, Yang RJ (2007) Three-dimensional hydrodynamic focusing in two-layer polydimethylsiloxane (PDMS) microchannels. J Micromech Microeng 17:1479-1486

Cheng IF, Chang HC, Hou D, Chang HC (2007) An integrated dielectrophoretic chip for continuous bioparticle filtering, focusing, sorting, trapping, and detecting. Biomicrofluidics 1:021503

Choi C, Park JK (2008) Sheathless Hydrophoretic particle focusing in a microchannel with exponentially increasing obstacle arrays. Anal Chem 80:3035-3039

Choi S, Song S, Choi C, Park JK (2007) Sheathless focusing of microbeads and blood cells based on hydrophoresis. Small 4:634-641

Chung TD, Kim HC (2007) Recent advances in miniaturized microfluidic flow cytometry for clinical use. Electrophoresis 28:4511-4520

Cummings EB, Singh AK (2003) Dielectrophoresis in Microchips Containing Arrays of Insulating Posts: Theoretical and Experimental Results. Anal Chem 75:4724-4731

Di Carlo D, Irimia D, Tompkins RG, Toner M (2007) Continuous inertial focusing, ordering, and separation of particles in microchannels. Proc Natl Acad Sci 104:18892-18897

Di Carlo D, Edd JF, Irimia D, Tompkins RG, Toner M (2008) Equilibrium separation and filtration of particles using differential inertial focusing. Anal Chem 80:2204-2211

Duffy DC, McDonald JC, Schueller OJA, Whitesides GM (1998) Rapid prototyping of microfluidic systems in poly(dimethylsiloxane). Anal Chem 70:4974-4984

Ermolina I, Morgan H (2005) The electrokinetic properties of latex particles: comparison of electrophoresis and dielectrophoresis. J Colloid Interf Sci 285:419-428

Fu LM, Yang RJ, Lee GB (2003) Electrokinetic focusing injection methods on microfluidic devices. Anal Chem 75:1905-1910

Hawkins BG, Smith AE, Syed YA, Kirby BJ (2007) Continuous-flow particle separation by 3D insulative dielectrophoresis using coherently shaped, DC-biased, AC electric fields. Anal Chem 79:7291-7300

Huh D, Gu W, Kamotani Y, Grotgerg JB, Takayama S (2005) Microfluidics for flow cytometric analysis of cells and particles. Physiol Meas 26:R73-R98

Kang K, Kang Y, Xuan X, Li D (2006a) Continuous separation of microparticles by size with DC-dielectrophoresis. Electrophoresis $27: 694-702$

Kang K, Xuan X, Kang Y, Li D (2006b) Effects of the DCdielectrophoretic force on particle trajectories in microchannels. J Appl Phys 99:064702

Kohlheyer D, Unnikrishnan S, Besselink GAJ, Schlautmann S, Schasfoort RBM (2008) A microfluidic device for array patterning by perpendicular electrokinetic focusing. Microfluid Nanofluid 4:557-564

Lee GB, Chang CC, Huang SB, Yang RJ (2006) The hydrodynamic focusing effect in rectangular microchannels. J Micromech Microeng 16:1024-1032

Lewpiriyawong N, Yang C, Lam YC (2008) Dielectrophoretic manipulation of particles in a modified microfluidic $\mathrm{H}$ filter with multi-insulating blocks. Biomicrofluidics 2:034105
Lin CH, Lee GB, Fu LM, Hwey BH (2004) Vertical focusing device utilizing dielectrophoretic force and its application on microflow cytometer. J Microelectromech Syst 13:923-932

Mao XL, Waldeisen JR, Huang TJ (2007) "Microfluidic drifting"implementing three-dimensional hydrodynamic focusing with a single-layer planar microfluidic device. Lab Chip 7:1260-1262

Morgan H, Green NG (2002) AC Electrokinetic: Colloids and Nanoparticles. Research Studies Press, Hertfordshire, UK

Pamme N (2007) Continuous flow separations in microfluidic devices. Lab Chip 7:1644-1659

Santiago JG (2001) Electroosmotic flows in microchannels with finite inertial and pressure forces. Anal Chem 73:2353-2365

Seo J, Lean MH, Kole A (2007a) Membrane-free microfiltration by asymmetric inertial migration. Appl Phys Lett 91:033901

Seo J, Lean MH, Kole A (2007b) Membraneless microseparation by asymmetry in curvilinear laminar flows. J Chromatogr A 1162:126-131

Shi J, Mao X, Ahmed D, Colletti A, Huang TJ (2008) Focusing microparticles in a microfluidic channel with standing surface. Lab Chip 8:221-223

Shilov VN, Delgadob AV, González-Caballero F, Horno J, LópezGarcía JJ, Grosse C (2000) Polarization of the electrical double layer. Time evolution after application of an electric field. J Colloid Interf Sci 232:141-148

Simonnet C, Groisman A (2006) High-throughput and high-resolution flow cytometry in molded microfluidic devices. Anal Chem 78:5653-5663

Takahashi T, Ogata S, Nishizawa M, Matsue T (2003) A valveless switch for microparticle sorting with laminar flow streams and electrophoresis perpendicular to the direction of fluid stream. Electrochem Commun 5:175-177

Thamida SK, Chang HC (2002) Nonlinear electrokinetic ejection and entrainment due to polarization at nearly insulated wedges. Phys Fluid 14:4315-4328

Thwar PK, Linderman JJ, Burns MA (2007) Electrodeless direct current dielectrophoresis using reconfigurable field-shaping oil barriers. Electrophoresis 28:4572-4581

Tornay R, Braschler T, Demierre N, Steitz B et al (2008) Dielectrophoresis-based particle exchanger for the manipulation and surface functionalization of particles. Lab Chip 8:267-273

Tsai CG, Hou HH, Fu LM (2008) An optimal three-dimensional focusing technique for micro-flow cytometers. Microfluid Nanofluid 5:827-836

Voldman J (2006) Electrical forces for microscale cell manipulation. Annu Rev Biomed Eng 8:425-454

Xuan X, Li D (2005) Focused electrophoretic motion and selected electrokinetic dispensing of particles and cells in cross-microchannels. Electrophoresis 26:3552-3560

Xuan X, Xu B, Li D (2005) Accelerated particle electrophoretic motion and separation in converging-diverging microchannels. Anal Chem 77:4323-4328

Xuan X, Raghibizadeh S, Li D (2006) Wall effects on electrophoretic motion of spherical polystyrene particles in a rectangular poly (dimethylsiloxane) microchannel. J Colloid Interf Sci 296:743748

Yang RJ, Chang CC, Huang SB, Lee GB (2005) A new focusing model and switching approach for electrokinetic flow inside microchannels. J Micromech Microeng 15:2141-2148

Yu C, Vykoukal J, Vykoukal DM, Schwartz JA et al (2005) A threedimensional dielectrophoretic particle focusing channel for microcytometry applications. J Microelectromech Syst 14:480-487

Zhao Y, Fujimoto BS, Jeffries GDM, Schiro PG, Chiu DT (2007) Optical gradient flow focusing. Opt Express 15:6167-6176 